\title{
Incorporation of Alkali Lignin in Polyacrylonitrile: Phase Separation, Coagulation and Cyclization Kinetics
}

Qian He ${ }^{a, c}$, Pucha Zhou ${ }^{a, d}$, Jian Hao ${ }^{a, c}$, Chunxiang Lu $u^{a, b, d^{*}}$, Yaodong Liu Lib,d* $^{*}$

${ }^{a}$ Key Laboratory of Carbon Materials, Institute of Coal Chemistry, Chinese Academy of Sciences, 27 Taoyuan South Road, Taiyuan 030001 China

${ }^{\mathrm{b}}$ Center of Materials Science and Optoelectronics Engineering, University of Chinese Academy of Sciences, Beijing 100049, China

c University of Chinese Academy of Sciences, 19 Yuquan Road, Beijing 100049, China d National Engineering Laboratory for Carbon Fiber Technology, Institute of Coal Chemistry, Chinese Academy of Sciences, 27 Taoyuan South Road, Taiyuan 030001, China Corresponding author: Y.Liu (1iuyd@sxicc.ac.cn); C.Lu (hunxl@sxicc.ac.cn)

KEYWORDS: Polyacrylonitrile, Alkali lignin, Phase separation, Film, Stabilization 
Table of Contents

1. SEM images of AL powder

2. Rheological data of PAN/AL solutions

3. EDS result of PAN/AL composite films

4. Frequency dependent Tan $(\delta)$ versus temperature curves $\quad \mathrm{S} 6$

$\begin{array}{ll}\text { 5. Peak fitting analysis of DSC curves } & \text { S7 }\end{array}$

6. TGA and differential curves of AL powder S8

7. DSC curve of AL powder $\quad 59$ 


\section{SEM images of AL powder}

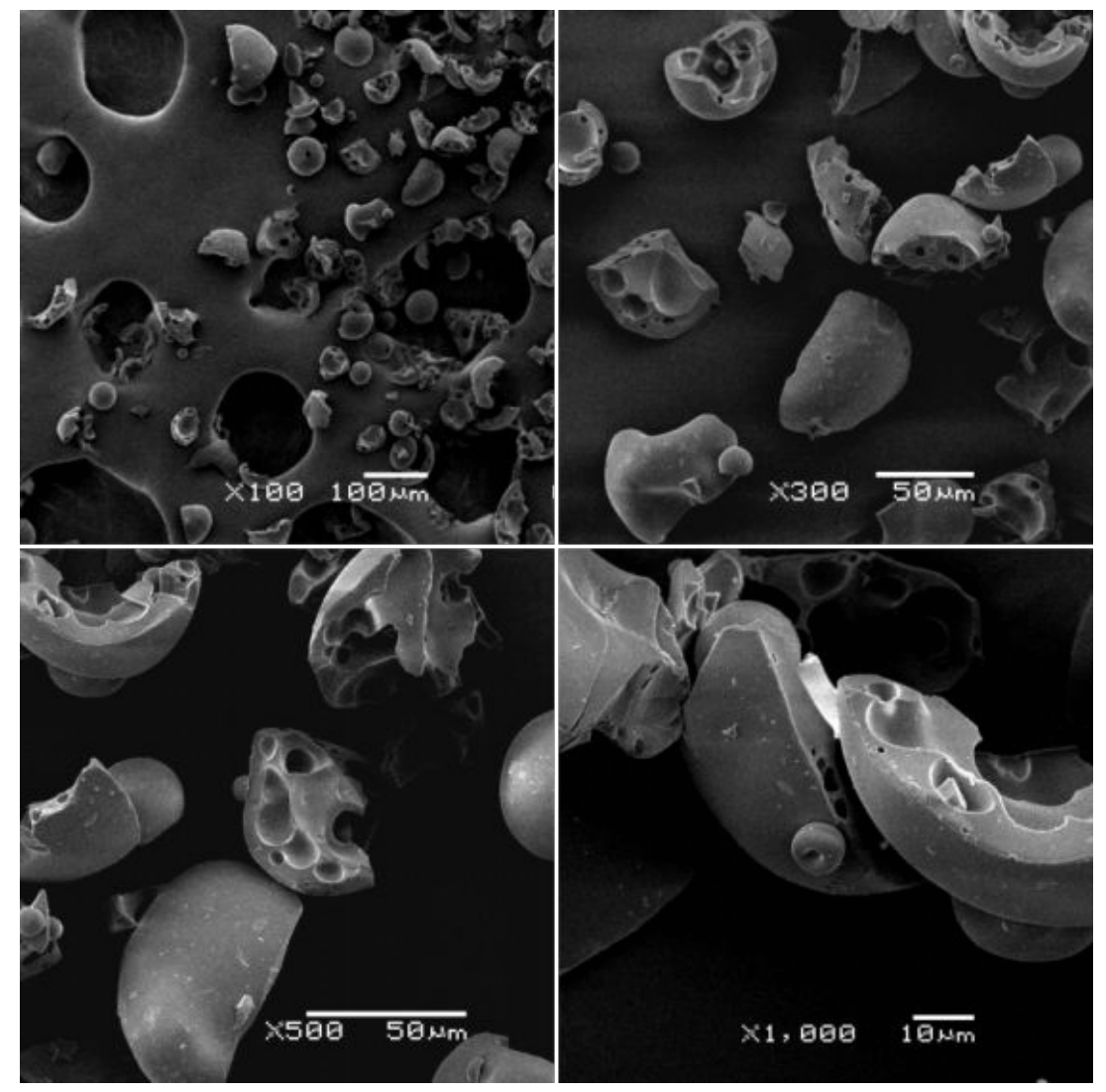

Figure S1. SEM images of as-received AL powder at various magnifications. 


\section{Rheological data of PAN/AL solutions}

Table S1. Summarized rheological data of PAN/AL solutions

\begin{tabular}{|c|c|c|c|c|c|}
\hline & \multicolumn{2}{|l|}{$\mathrm{PAN} / \mathrm{AL} / \mathrm{DMSO}$} & Sol-PAN & Sol-AL2 & Sol-AL5 \\
\hline \multirow{4}{*}{ Fresh } & $\mathrm{n}=\mathrm{n} 1 / \mathrm{n} 2$ & & 1.88 & 1.78 & 1.74 \\
\hline & complex viscosity $(\mathrm{Pa}$ & - s) & 59 & 25 & 52 \\
\hline & \multirow{2}{*}{ Gelation Temperature $\left({ }^{\circ} \mathrm{C}\right)$} & Cooling & 9.3 & 6.5 & 4.4 \\
\hline & & Heating & 13.4 & 13.1 & 13.1 \\
\hline \multirow{4}{*}{ Aged - } & $\mathrm{n}=\mathrm{n} 1 / \mathrm{n} 2$ & & 1.90 & 1.85 & 1.88 \\
\hline & complex viscosity $(\mathrm{Pa}$ & • $\mathrm{s})$ & 61 & 29 & 57 \\
\hline & \multirow{2}{*}{ Gelation Temperature $\left({ }^{\circ} \mathrm{C}\right)$} & Cooling & 9.0 & 5.0 & 3.5 \\
\hline & & Heating & 13.5 & 13.0 & 13.0 \\
\hline
\end{tabular}


3. EDS result of PAN/AL composite films

Table S2. EDS: atom percentages

\begin{tabular}{cccc}
\hline Position & 1 & 2 & 3 \\
\hline $\mathrm{C}$ & $58 \%$ & $57 \%$ & $58 \%$ \\
$\mathrm{~N}$ & $15 \%$ & $23 \%$ & $0 \%$ \\
$\mathrm{O}$ & $17 \%$ & $20 \%$ & $42 \%$ \\
\hline
\end{tabular}




\section{Frequency dependent Tan $(\delta)$ versus temperature curves}
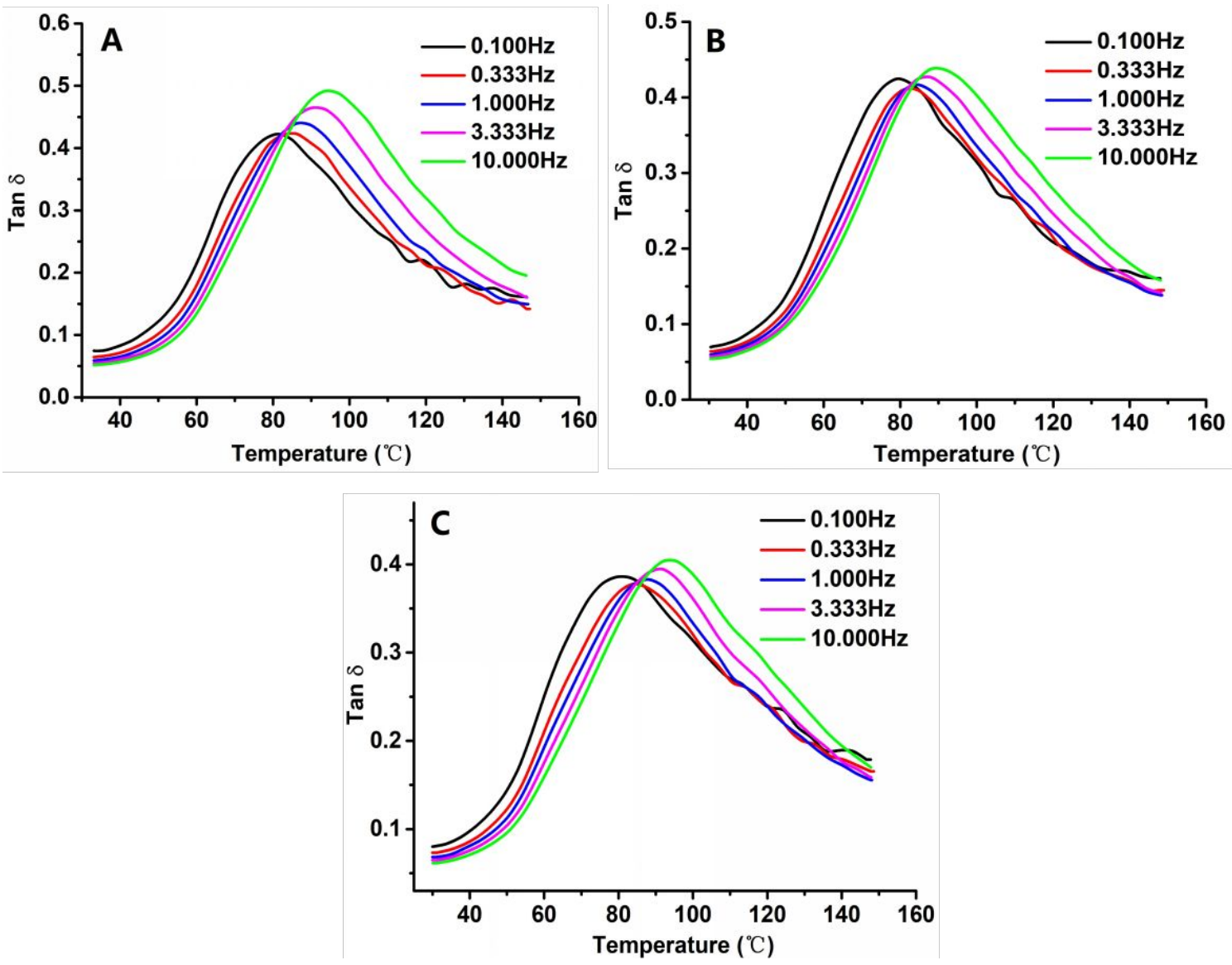

Figure S2. Frequency dependent Tan $(\delta)$ versus temperature curves for PAN and PAN/AL composite films (A: Film-C-P, B: Film-C-AL2, and C: Film-CAL5). 


\section{Peak fitting analysis of DSC curves}
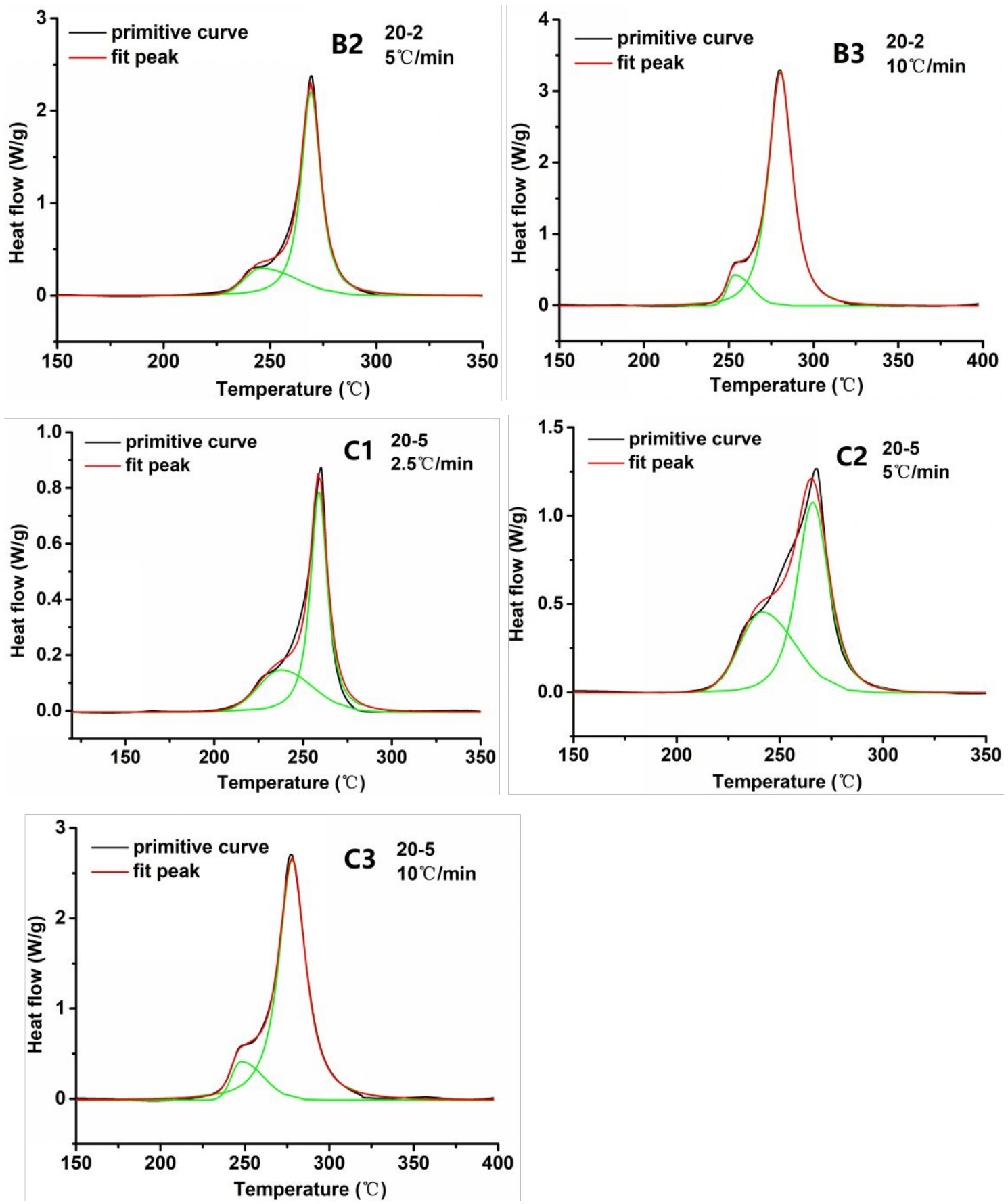

Figure S3. Peak fitting analysis of DSC curve for PAN/AL composite films at different heating rates: Bigaussian function for peak 1 (main peak) and PearsonVII function for peak 2 (sub-peak) (B2-B3, C1-C3). 
6. TGA and differential curves of AL powder

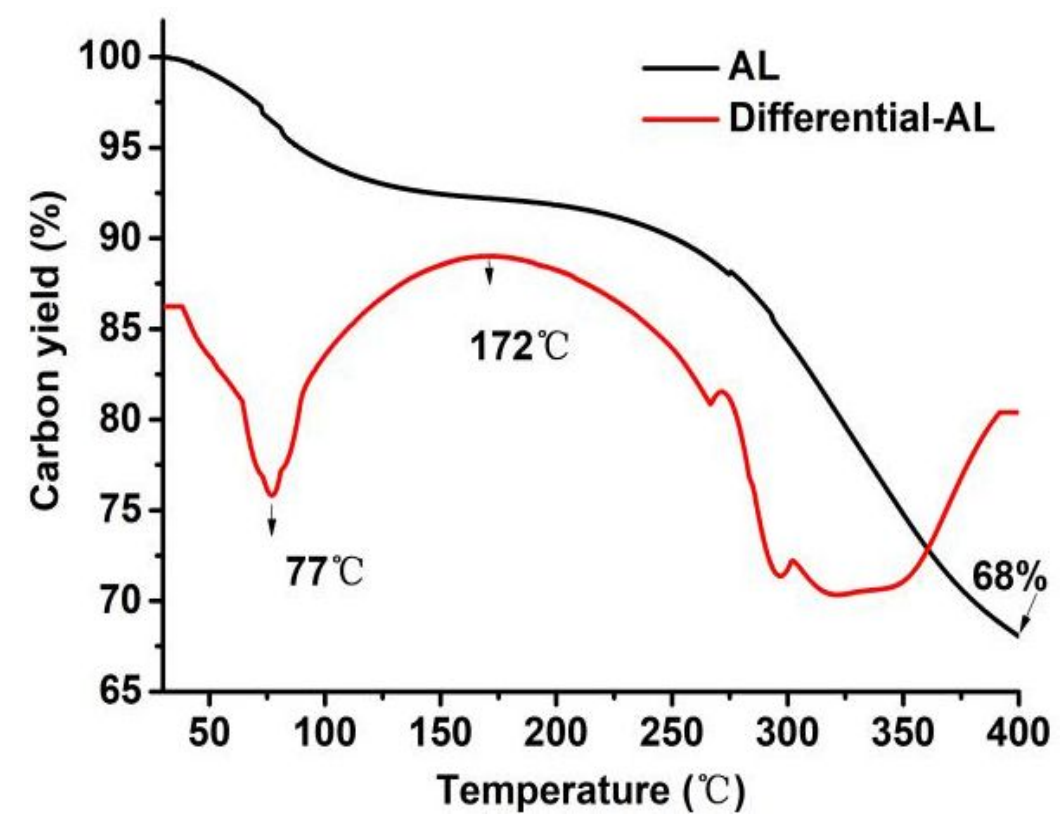

Figure S4. TGA and differential curves of AL powder under $\mathrm{N}_{2}$ atmosphere up to $400{ }^{\circ} \mathrm{C}$. 
7. DSC curve of AL powder

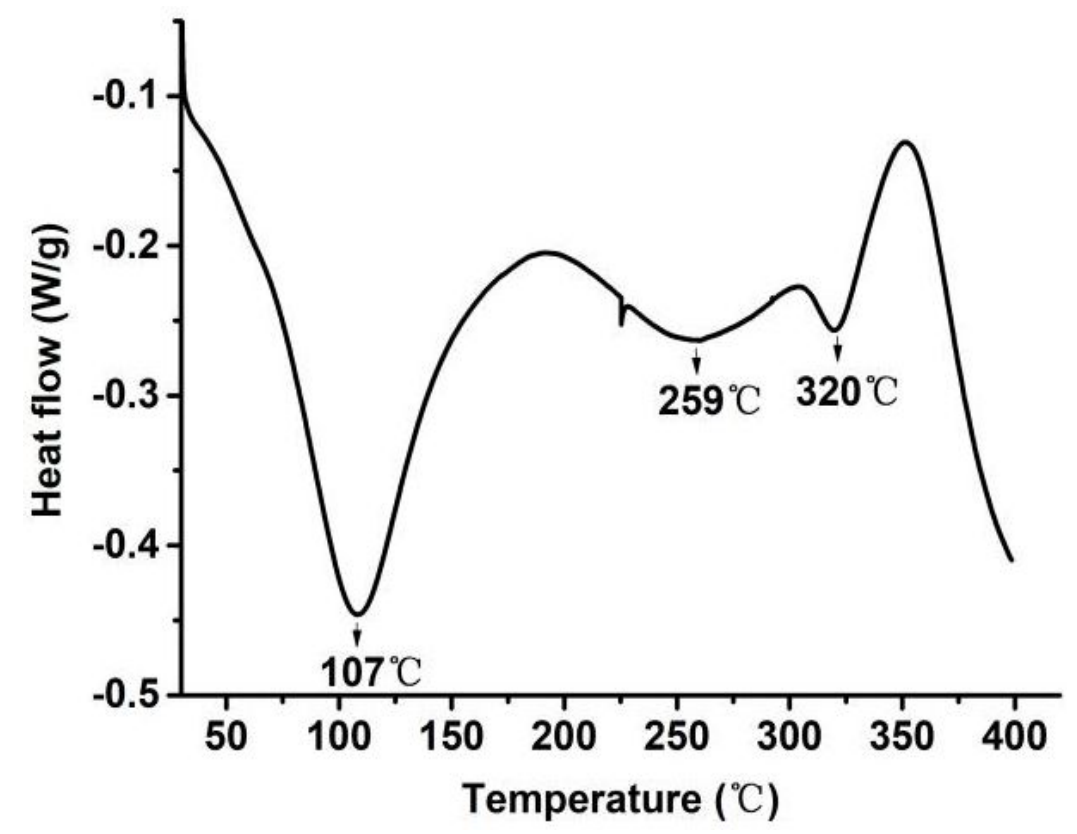

Figure S5. DSC curve of AL powder under $\mathrm{N}_{2}$ atmosphere up to $400{ }^{\circ} \mathrm{C}$. 